\title{
Lung Hernia Associated With Hemothorax Following Cardiopulmonary Resuscitation
}

\author{
John S Emberger Jr RRT FAARC, Lisa Racine RRT, and Vinay Maheshwari MD
}

\begin{abstract}
Lung herniation is a rare event that can occur spontaneously or traumatically. Thoracic hernias are usually associated with a chest-wall defect. We report a case of thoracic lung hernia that occurred $\mathbf{2}$ days after traumatic cardiopulmonary resuscitation, after the formation of a large hemothorax. Key words: lung herniation; cardiopulmonary resuscitation; hemothorax. [Respir Care 2011;56(7):10371039. () 2011 Daedalus Enterprises]
\end{abstract}

\section{Introduction}

Lung hernia is an uncommon clinical occurrence. Lung hernia is defined as the protrusion of the lung through one of its bounding structures. ${ }^{1}$ Lung hernias are classified according to location (cervical, thoracic, or diaphragmatic) or cause (congenital, traumatic, or spontaneous). Thoraciclocation lung hernias are most common and are highly associated with trauma. ${ }^{1,2}$ We report a case of a thoracic lung hernia associated with fractured ribs and hemothorax.

\section{Case Report}

A 69-year-old male with a history of COPD was found apneic and pulseless by paramedics. Soon after cardiopulmonary resuscitation was initiated, circulation returned, and he was intubated and mechanically ventilated upon arrival at the emergency department. He was admitted to the medical intensive care unit and underwent our hypothermia protocol for post-cardiac-arrest care. Computed tomogram on day 2 showed fractures of the anterior right third, fourth, and fifth ribs, and the anterior left fourth and fifth ribs. Chest radiographs during the first 3 days showed no active cardiopulmonary disease processes. He remained

The authors are affiliated with the Department of Respiratory Care, Christiana Care Health System, Newark, Delaware.

The authors have disclosed no conflicts of interest.

Correspondence: John S Emberger Jr RRT FAARC, Department of Respiratory Care, Christiana Care Health System, 4755 Ogletown-Stanton Road, Newark DE 19701. E-mail: jemberger@christianacare.org.

DOI: $10.4187 /$ respcare.01069 intubated and mechanically ventilated on volume assist control ventilation. On day 3 a large left pleural effusion was noted on the morning chest radiograph (Fig. 1A). A follow-up computed tomogram was performed and a chest tube was placed. In addition to confirming the pleural fluid, the computed tomogram revealed herniation of lung through the 4th rib fracture, and a large hemothorax of the left lung (Fig. 2). The chest tube drained 1,200 mL of blood. His physiologic response included increased heart rate and worsening ventilation pressures and mechanics (Fig. 3).

Chest radiograph confirmed that the pleural effusion was resolving (see Fig. 1B), and the chest tube and lung hernia had resolved as well. There was no recurrence of the lung herniation during the next 15 days before discharge (Fig. 4). He underwent tracheotomy on day 12, and on day 15 he was liberated from the ventilator and discharged to a subacute nursing facility with tracheostomy tube for airway protection, due to anoxic encephalopathy.

\section{Discussion}

Herniation of the lung is an uncommon condition that was first described in 1499, by Roland. ${ }^{1}$ Diagnosis is made with chest radiograph or CT. Lung herniation was further described by Morel-Lavelle, with classifications based on etiology (acquired versus congenital) and anatomical location (cervical or thoracic). Acquired lung hernias most commonly are due to blunt or penetrating trauma, or following chest-wall surgery. ${ }^{2-9}$ Lung hernias have also been described as a result of tumors, chest-wall abscess, or invasion from tuberculosis. ${ }^{9}$ Spontaneous lung hernias have been described following vigorous coughing episodes, but are often at sites of previous surgery or intervention. . $^{3,6-8}$ 

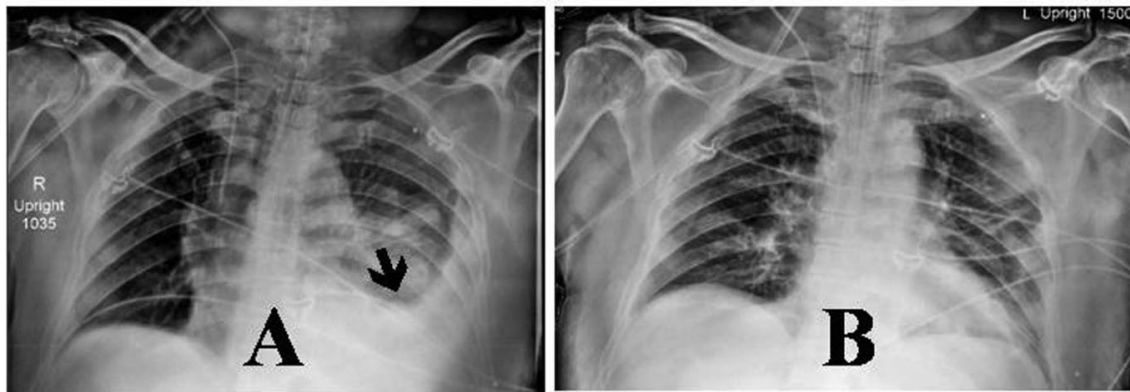

Fig. 1. Chest radiograph before $(A)$ and after $(B)$ chest tube placement for a large hemothorax (arrow) causing thoracic lung herniation through rib fractures.

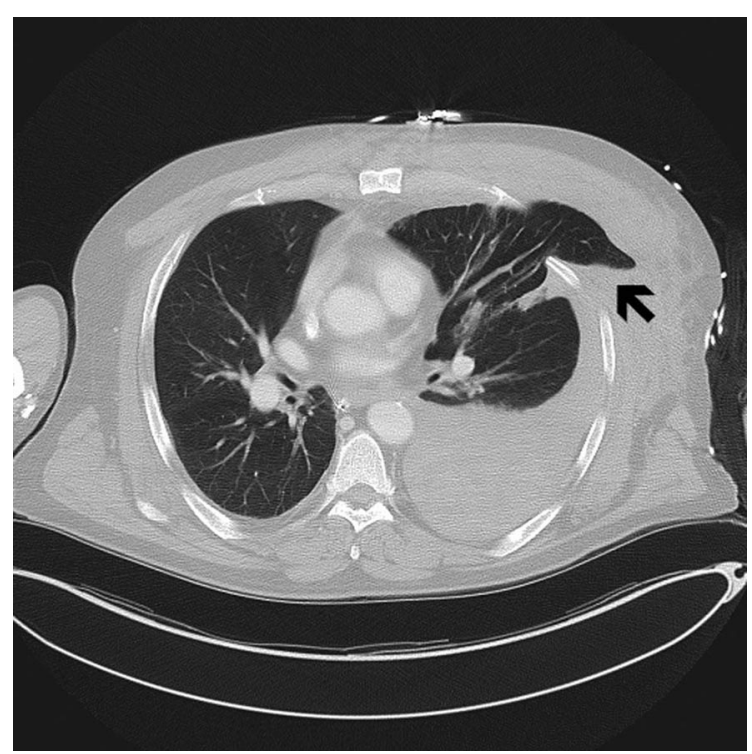

Fig. 2. Computed tomogram shows the left hemothorax and resulting thoracic lung herniation (arrow).

Acquired lung hernias typically occur soon after the inciting event, but delays of years have also been described.

Both conservative and surgical management of lung hernias have been described.6,9-11 If the patient is asymptomatic and has a supraclavicular hernia, no intervention may be necessary. However, surgical intervention may be needed if there is increasing size, pain, or signs of incarceration. Cosmetic reasons have also been cited as reasons to consider surgical repair. A variety of surgical procedures have been described, most of which include both autologous tissue, such as muscle or fascia layers, and synthetic mesh layers. ${ }^{9,10}$ Recently a lung hernia procedure was described that involves laminar hooks, which are typically used in managing spinal injuries. ${ }^{11}$

Our patient demonstrated lung herniation at the site of traumatic cardiopulmonary resuscitation. The lung hernia did not become apparent until a large hemothorax developed from rib-fracture injury of an intercostal artery. With

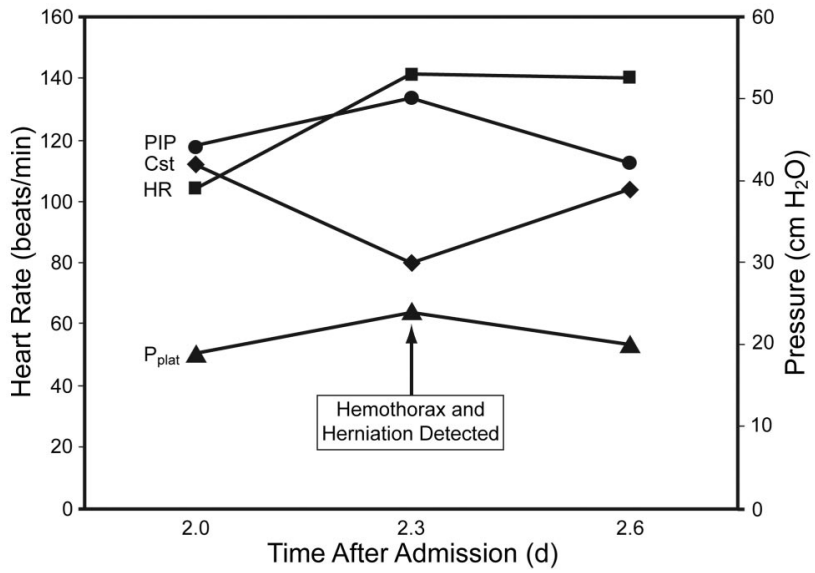

Fig. 3. Response of heart rate and ventilator pressures to the hemothorax causing lung herniation. $\mathrm{PIP}=$ peak inspiratory pressure. $\mathrm{P}_{\text {plat }}=$ plateau pressure. $\mathrm{C}_{\mathrm{st}}=$ lung compliance.

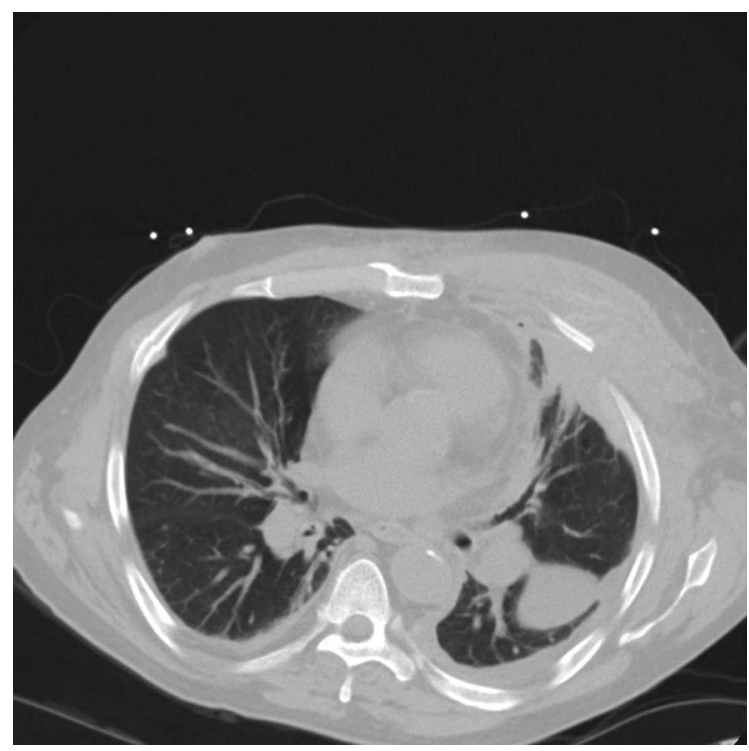

Fig. 4. Computed tomogram shows reduced hemothorax and resolution of thoracic lung herniation on day 15. 


\section{Lung Hernia Associated With Hemothorax Following Cardiopulmonary Resuscitation}

drainage of the hemothorax the lung hernia resolved and further surgical intervention was not required.

\section{REFERENCES}

1. Montgomery JG, Lutz H. Hernias of the lung. Ann Surg 1925;82(2): 220-231.

2. Tack D, Wattiez A, Schtickzelle JC, Delcour C. Spontaneous lung herniation after a single cough. Eur Radiol 2000;10(3):500-502.

3. DiMarco AF, Oca O, Renston JP. Lung herniation: a cause of chronic chest pain following thoracotomy. Chest 1995;107(3):877-879.

4. Sulaiman A, Cottin V, De Souza Neto EP, Orsini A, Cordier JF, Gamondes JP, Tronc F. Cough-induced intercostal lung herniation requiring surgery: report of a case. Surg Today 2006;36(11):978-980.

5. Athanassiadi K, Bagaev E, Simon A, Haverich A. Lung herniation: a rare complication in minimally invasive cardiothoracic surgery. Eur J Cardiothorac Surg 2008;33(5):774-776.
6. Rhodes CM, Gregory J. Complete thoracic cage disruption associated with anterior lung herniation. J Trauma 2009;67(5):E146E148.

7. Bhalla M, Leitman BS, Forcade C, Stern E, Naidich DP, McCauley DI. Lung hernia: radiographic features. AJR Am J Roentgenol 1990; 154(1):51-53.

8. Lang-Lazdunski L, Bonnet PM, Pons F, Brinquin L, Jancovici R. Traumatic extrathoracic lung herniation. Ann Thorac Surg 2002; 74(3):927-929.

9. Weissberg D, Refaely Y. Hernia of the lung. Ann Thorac Surg 2002;74(6):1963-1966.

10. Szentkereszty Z, Boros M, Sapy P, Kiss SS. Surgical treatment of intercostal hernia with implantation of polypropylene mesh. Hernia 2006;10(4):354-356.

11. Wiens S, Hunt I, Mahood J, Valji A, Stewart K, Bedard ELR. Novel fixation technique for the surgical repair of lung hernias. Ann Thorac Surg 2009;88(3):1034-1035. 